\title{
Post-fatigue Investigation of SLM Ti64 Scaffolds by 3D Correlative Tomography
}

Bartlomiej Winiarski ${ }^{1,2}$, Michelle Dallago ${ }^{3}$, Matteo Benedetti ${ }^{3}$ and Philip J. Withers ${ }^{2}$

${ }^{1}$ Thermo Fisher Scientific, Brno, Jihomoravsky kraj, Czech Republic, ${ }^{2}$ The Henry Royce Institute, School of Materials, The University of Manchester, Manchester, England, United Kingdom, ${ }^{3}$ University of Trento, Trento, Lombardia, Italy,

*Corresponding author: Bartlomiej.Winiarski@thermofisher.com

Open-porous Ti64 scaffolds or foams [1] are attractive in the field of biomedical implants, since these show reduced stress-shielding and improved osseointegration [2]. Mechanical properties and geometric characteristics of implant scaffolds are tailored to match that of a patient's bone by using of computeraided design (CAD) and finite-element analysis (FEA). Later CAD-FEA designed implants are produced by means of various additive manufacturing (AM) techniques, e.g. selective laser melting of Ti64 (SLM) [3]. Nevertheless, several issues still exist to resolve, e.g. detrimental residual stress states, mechanical properties far scattered from the FEA-predicted and the inconsistency between the CAD-designed and the manufactured geometries. Ti-alloys in general have high notch sensitivity [4]. Surface roughness/notches and material defects are inherent feature of any AM process. These act as stress raisers and crack initiators, and strongly affect the fatigue resistance of an AM build [5].

In the previous study [3], the results of fatigue and quasi-static tests of hot isostatic pressed (HIP) Ti64 scaffold were compared with FE calculations based on the as-designed geometry of a regular cubic cellular scaffold and on the as-built geometry reconstructed from $\mu \mathrm{CT}$ scans. Both the elastic modulus and the fatigue resistance resulted strongly correlated with the number and severity of defects. Moreover, predictions of the mechanical properties based only on the as-designed geometry were shown not to be accurate [3].

The current study investigates the fatigued and fractured HIPed Ti64 cubic cellular scaffold (from study in [3]) using a 3D correlative multi-scale, multi-modal tomography and microscopy (CMT) framework (Figure 1). The CMT workflow combines advantages of imaging, analytical and metrological capabilities of various instruments and cross-platform correlative holder kit: helical micro X-ray computed tomography, plasma focused ion beam - scanning electron microscope (PFIB-SEM), Laser PFIB-SEM (where three beams are in a coincidence point) and other apparatus, e.g. transmission electron microscopes. The correlative approach investigates multiple fracture zones (FZ) and volumes in the vicinity of FZ in search for the weakest link. Brittle, ductile and mixed-mode fracture occurred in the locations having the smallest cross-sectional area of struts. The type of fracture of each strut strongly depends on the crystallographic orientation of $\alpha$ and $\beta$ phases and increased presence of brittle $\alpha$ phase.

Our correlative solution is supported by Maps-based [6] and Avizo-based workflows [7] and is compatible with the inert gas transfer hardware and the cryo stage. 

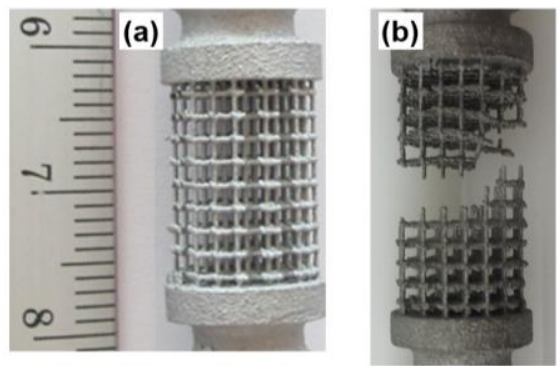

(g) Full sample $\mu \mathrm{CT}$ scan
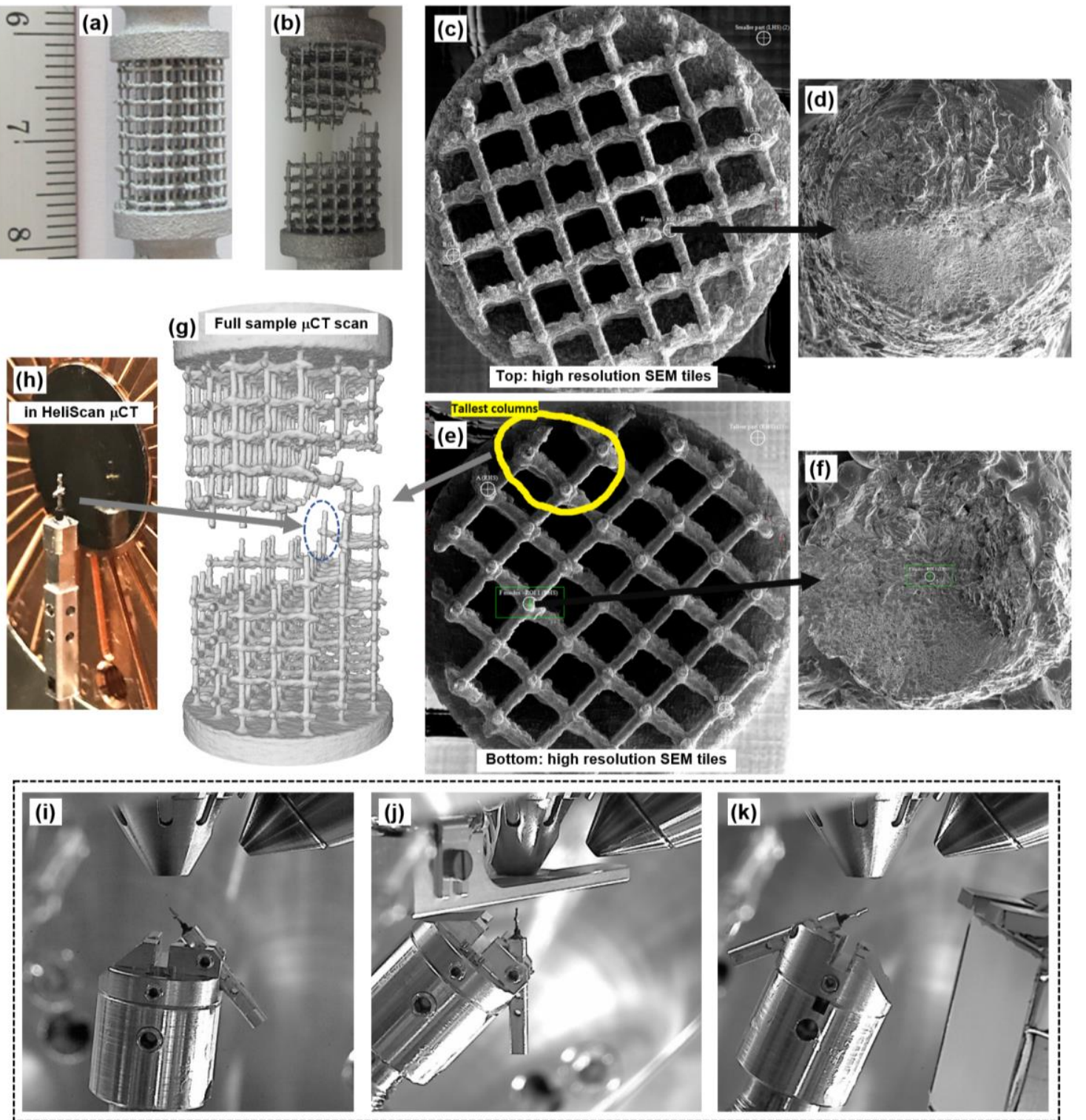

Figure 1. Shows post-fatigue investigation of Ti64 scaffolds by 3D correlative tomography. (a) as build and HIPed and (b) fatigued and fractured scaffold; (c) - (f) fractography study using tiled high resolution SEM images in Maps; (g) - (h) HeliScan $\mu \mathrm{CT}$ study using cross-platform correlative holder; (i) - (k) serial sectioning and EBSD study in Plasma FIB-SEM microscope using cross-platform correlative holder kit.

\section{References}

[1] R Singh, at al. Mater Tech Adv Perf Mater 2013; p. 127-136.

[2] LE Murr, at al. Philos Trans R Soc A Math Phys Eng Sci 2010; 368:1999-2032.

[3] M Dallago, B Winiarski, at al. International Journal of Fatigue 124 (2019) 348-360.

[4] M Niinomi. J Mech Behav Biomed Mater 2008;1:30-42.

[5] S Leuders, at al. Int J Fatigue 2013;48:300-7.

[6] B Winiarski, at al. Microscopy and Microanalysis 25(S2)-2019:870-871.

[7] B Winiarski, at al. Supplement of Microscopy and Microanalysis 152 (2017), p. S4-S9. 